Comm. in Asteroseismology

Vol. 153, 2008

\title{
The amplitudes of the $\delta$ Scuti star 4 CVn in 1991
}

\author{
M. Breger ${ }^{1}$, K. A. Davis ${ }^{2}$, and R. J. Dukes ${ }^{2}$ \\ ${ }^{1}$ Institut für Astronomie, Türkenschanzstr. 17, A-1180 Wien, Austria \\ ${ }^{2}$ College of Charleston, 66 George Street, Charleston SC 29424, USA
}

\begin{abstract}
The star $4 \mathrm{CVn}$ shows strong amplitude variability from year to year with an approximate range of a factor of two for each mode. 32 nights of photometry from 1991 are used to derive the photometric amplitudes of the ten dominant modes. The amplitude variability of all these modes is confirmed with time scales of years, but not decades. The amplitudes lie within the range for each frequency determined during previous studies of $4 \mathrm{CVn}$ covering 40 years.
\end{abstract}

Individual Object: $4 \mathrm{CVn}$

\section{Introduction and Observations}

The variability of $4 \mathrm{CVn}$ was discovered by Jones \& Haslam (1966). It is interesting that in the late 1960s, the dominant modes of $4 \mathrm{CVn}$ had frequencies of 5.048 and $5.851 \mathrm{c} / \mathrm{d}$, while later measurements seemed to disagree due to the newly dominant modes with completely different frequencies of 8.585 and $7.375 \mathrm{c} / \mathrm{d}$.

We now know that $4 \mathrm{CVn}$ is an evolved $\delta$ Scuti pulsator exhibiting an extreme amount of amplitude variability with a time scale of years (Breger 2000). This is in contrast to more rapid amplitude variability seen in several other $\delta$ Scuti stars such as $\mathrm{BI} \mathrm{CMi}$, which has been shown to be caused by the beating between close frequencies. To understand the origin of the slow amplitude variability, it is essential to analyze measurements covering many decades. The situation is made more complicated by the fact that these stars pulsate in a large number of photometrically detectable modes, requiring extensive campaigns to study individual pulsation modes. For $4 \mathrm{CVn}$, data from 1966 to 2007 are available. These data, however, contain long time gaps, e.g., between 1984 and 1996. 
Table 1: Photometric amplitudes of 4 CVn in 1991

\begin{tabular}{ccc}
\hline $\begin{array}{r}\text { Frequency } \\
(\mathrm{c} / \mathrm{d})\end{array}$ & $\begin{array}{c}\text { Semi-amplitude } \\
\text { mag }\end{array}$ \\
\hline$f_{1}$ & 8.595 & 0.0106 \\
$f_{2}$ & 7.375 & 0.0162 \\
$f_{3}$ & 5.048 & 0.0094 \\
$f_{4}$ & 6.117 & 0.0104 \\
$f_{5}$ & 5.851 & 0.0115 \\
$f_{6}$ & 5.532 & 0.0148 \\
$f_{7}$ & 6.190 & 0.0098 \\
$f_{8}$ & 6.976 & 0.0086 \\
$f_{9}$ & 7.552 & 0.0084 \\
$f_{10}$ & 6.752 & 0.0065 \\
\hline
\end{tabular}

The gap around 1990 can be bridged by the previously unpublished observations by one of us (Dukes). The star was observed during 1991 using the $0.75 \mathrm{~m}$ Four College Consortium Automatic Photometric Telescope on Mt. Hopkins, Arizona, USA, through a Stromgren $b$ filter. A preliminary report of this was given by Davis \& Dukes (2001).

A comparison star, HD 108100, and a check star, HD 111834, were used. Regrettably, HD 108100 has turned out to be a small-amplitude Gamma Doradus variable (Breger et al. 1997). Consequently, the photometry of $4 \mathrm{CVn}$ was reduced relative to the check star only. Because of this, normal quality control measures, which involve rejecting observations whose check minus comparison values are greater than 3 standard deviations from the mean value, could not be used. Thus, instead of assuming a constant check minus comparison value, we fitted the two frequencies found for HD 108100 by Breger et al. (1997) and required that each check minus comparison value be less than 3 standard deviations from this fit. 598 photometric measurements obtained during 32 nights were used. Problems with a variable zero-point from night to night between the two stars could not be resolved so that the various nights were joined by applying zero-point shifts optimized for lowest residuals. Such an approach ruins the low-frequency information, which is unimportant for $4 \mathrm{CVn}$.

The data are well-suited to derive accurate amplitudes of the well-known dominant modes. The campaign covered a time period of 83 nights, leading to a frequency resolution of $0.03 \mathrm{c} / \mathrm{d}$ or better.

\section{Multifrequency analyses}

The pulsation frequency analyses were performed with a package of computer programs with single-frequency and multiple-frequency techniques (PERIOD04, Lenz \& Breger 2005), which utilize Fourier as well as multiple-least-squares algorithms. The 
latter technique fits up to several hundreds of simultaneous sinusoidal variations in the magnitude domain and does not rely on sequential prewhitening. The amplitudes and phases of all modes/frequencies are determined by minimizing the residuals between the measurements and the fit. We restricted ourselves to frequencies greater than $3 \mathrm{c} / \mathrm{d}$, in which the main pulsation modes of $4 \mathrm{CVn}$ were found. The uncertainties in the amplitudes were determined from a number of Monte Carlo simulations in $\mathrm{Pe}-$ riod04 to be $\pm 0.0010 \mathrm{mag}$. This number is a factor of two higher than the value calculated with the formal equations given in Breger et al. (1999).

$4 \mathrm{CVn}$ has at least 42 known frequencies of pulsation. This means that for a medium-size campaign, overinterpretation of the data is possible. This might lead to different frequencies 'bumping' each other to higher amplitudes. Fortunately, most of the frequencies detected in $4 \mathrm{CVn}$ have amplitudes in the submillimag range. Nevertheless, to avoid this problem, we have adopted a very conservative approach: a full solution assuming all known frequencies of pulsation (together with their harmonics and combinations) was not made: only frequencies which were clearly visible in the Fourier spectra of the 1991 data were considered. This led to the rediscovery of the 10 frequencies shown in Table 1. Fortunately, these are also the dominant modes known from the more extensive studies undertaken in later years. This allowed us to adopt the previously known exact values of the frequencies.

The 10 frequencies shown in the table lead to an excellent solution with a fit good to \pm 0.007 mag per single measurement. Next, we have included the additional frequencies known to be excited in the star on a one-by-one basis. Only two modes $(6.680$ and $6.440 \mathrm{c} / \mathrm{d})$ showed amplitudes in excess of $0.001 \mathrm{mag}$ (i.e., 0.0015 and $0.0013 \mathrm{mag}$, respectively) and they may, therefore, also be real in the 1991 data with very small amplitudes. The amplitude-signal/noise ratio of detection of these two modes is only around 2 , which does not qualify as a significant detection.

\section{Discussion}

The 1991 data lie in the gap between the 1983/84 and 1995/96 measurements. The remaining gaps are still too large to answer the question of whether periodicities in the amplitude variations exist. To compare the amplitudes with those of other years, we have scaled the $b$ values by a factor of 0.86 to transform them to $V$. The factor was estimated from scaling the known Stromgren $v$ and $y$ amplitudes of $4 \mathrm{CVn}$.

A comparison of the amplitudes listed in Table 1 with the values from other years, including those of 2005-2007, allows the following conclusions:

i The amplitude variability of the various modes of $4 \mathrm{CVn}$ and the time scale of years, but not decades, are confirmed.

ii The 1991 data do not exhibit surprises or new dominant frequencies.

iii The amplitudes differ from those of the 1983/84 and 1995/96 observing seasons, but lie within the ranges previously found in the 40 years of data.

iv In particular, during 1991 the amplitude of $f_{1}$ is near its minimum, the amplitudes of $f_{2}, f_{4}, f_{6}, f_{7}, f_{9}$, and $f_{10}$ are near their maximum values, while $f_{3}$, $f_{5}$, and $f_{8}$ are near their average amplitude values. 
Acknowledgments. This investigation has been supported by the Austrian Fonds zur Förderung der wissenschaftlichen Forschung and the U.S. National Science Foundation.

\section{References}

Breger, M. 2000, MNRAS 313, 129

Breger, M., Handler, G., Garrido, R., et al. 1997, A\&A 324, 566

Breger, M., Handler, G., Garrido, R., et al. 1999, A\&A 349, 225

Davis, K. A., \& Dukes, R. J. 2001, BAAS 33, 1436

Jones, D. H. P., \& Haslam, C. M. 1966, Obs 86, 34

Lenz, P., \& Breger, M. 2005, CoAst 146, 53 